\title{
PROXIMAL PRIORITY TREATMENT USING THE NECK IRRADIATOR FOR ADJUNCTIVE TREATMENT OF FEMALE INFERTILITY
}

\author{
*Shunji Fujii M.D. ${ }^{1}$, Toshio Ohshiro M.D., Ph.D. ${ }^{2}$, \\ Takafumi Ohshiro M.D. ${ }^{1}$, Katsumi Sasaki M.D. ${ }^{1}$, and Yuki Taniguchi M.D. ${ }^{1}$ \\ 1: Ohshiro Clinic, Tokyo, Japan \\ 2: Japan Medical Laser Laboratory, Tokyo, Japan
}

\begin{abstract}
Recently in Japan, the trend towards females marrying later in life than before has increased the average age of women bearing children for the first time. The number of children being born in Japan has steadily decreased for the $26^{\text {th }}$ straight year and has become a grave socio-economical concern for the future. Artificial reproductive technology (ART) has shown advances in the past two decades, however there are no new solutions to the old problem of age. The authors have previously reported the efficacy of low reactive level laser therapy (LLLT) for the adjunctive treatment of refractory female infertility and will report a new method of LLLT, incorporating the Proximal Priority Treatment (PPT) with the use of novel device named the Neck Irradiator. A retrospective survey of patients receiving PPT with the Neck Irradiator showed that approximately 10\% of the patients became pregnant with this treatment. The authors compare and contrast this new treatment with previous treatment modalities and will discuss LLLT for the treatment of female infertility.
\end{abstract}

Key Words: LLLT, female infertility, poor responder, IVF

\section{Introduction}

The recent trend in Japan of females marrying later in life than was the case in previous times has led to an increase in the average age of women bearing children for the first time. The number of children being born in Japan has steadily decreased for the $26^{\text {th }}$ straight year and has become a grave socio-economical concern for the future. Artificial reproductive technology (ART) has shown advances in the past two decades but has been limited to advances in manipulation of gametes and embryos, such as intracytoplasmic sperm insemination, assisted hatching or to advances in incubation., while no cure for the problem of poor responders and the old problem of age has been presented. The authors have previously reported the use of low reactive level laser therapy (LLLT) for the treatment of infertility as an adjunct to ART where increased systemic blood flow increases the response of the ovary, hence increases the response to ART and increases the probability of preg-

Addressee for Correspondence:

Shunji Fujii M.D.

JR Shinanomachi-building 2F, 34 Shinanomachi, Shinjuku-

ku, Tokyo, Japan

160-0016

TEL: +81-3-3352-0046

FAX: +81-3-3354-1103

E-mail: info@jmll.co.jp nancy. The authors have tried to improve on the previously reported treatment method and devised what they term the proximal priority treatment (PPT). In addition, a new therapeutic device, the Neck Irradiator, was introduced to the treatment. The results over the conventional methods are compared and discussed.

\section{Patients and methods}

Infertile female patients presenting to Ohshiro clinic during the period of April 1, 2006 to January 31, 2007 were treated with the proximal priority treatment using the Neck Irradiator. Patients were placed in a supine position. Each treatment session consisted of 10 minutes of PPT where laser is irradiated solely to the nuchal area while the therapist first stretches the neck region and than goes on to stretch other areas in the order of shoulder, pectoral region, spine, lower back and finally the lower limbs. This treatment commences at the proximal region in relation to the two centers of the nervous system and circulatory system followed by treatment of the periphery, hence the name 'proximal priority treatment'. Another 10 minutes is allotted for local (abdominal-lumbar area) treatment for the stimulation of the reproductive organs for a total of 20 minutes of laser irradiation.

Manuscript received: June 2007

Accepted for publication: August 2007 


\section{ORIGINAL ARTICLES}

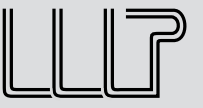

Patients received treatment once every one to two weeks, depending on the patients' schedule. Thirty-six patients were enrolled in this study and were retrospectively surveyed for any subjective and objectives changes throughout the treatment duration. Pregnancies, number of ART attempts and their results were recorded. Only patients receiving more than two treatments were included this study: others were considered drop-outs and were excluded from the final analysis.

\section{Laser Hardware}

The Neck Irradiator (Japan Medical Laser Laboratory Corp.) was used for the treatment (Fig. 1). The Neck Irradiator is a two channel GaAlAs diode laser device which emits laser light of $830 \mathrm{~nm}$ at an output of 60 $\mathrm{mW}$. Laser light is emitted from either the neck strap probe or from the hand piece, or from both. The device is therefore 'hands-free, thus allowing for less time consuming treatment sessions where the patient can concentrate on the treatment itself and the therapist can perform the necessary treatment maneuvers without having to work continuously with the hand piece.

\section{Results}

Since, April 1 1t, 200636 patients presented to Ohshiro clinic for treatment of infertility. Two patients were excluded from the study due to discontinuation of treatment after the initial session. Of the patients all but 2 were simultaneously being treated at infertility clinics and of those patients 30 had already attempted COS and IVF without success for an average of 6.6 times, and those remaining were still being treated by artificial insemination and were beginning to contemplate further treatments. The average age of the patients was 41.7 years, with ages ranging from 26 to 52 years and the average period of infertility was 6.58 years. Two patients were amenorrheic. Patients not attending infertility clinics did so due to personal beliefs or for economic reasons. No attempt from the clinic's part was taken to advise them otherwise. An average number of 10.2 LLLT sessions per patient was performed. Of the 34 patients 3 patients were documented for pregnancies during this time period for a pregnancy rate of $8.8 \%$ (Table 1).

\section{Discussion}

There are numerous causes of infertility, some of which may be treated with success while in others the cause itself is elusive with no means of treatment. Artificial reproductive procedures are merely methods of ensuring fertilization and subsequent transfer of the embryo

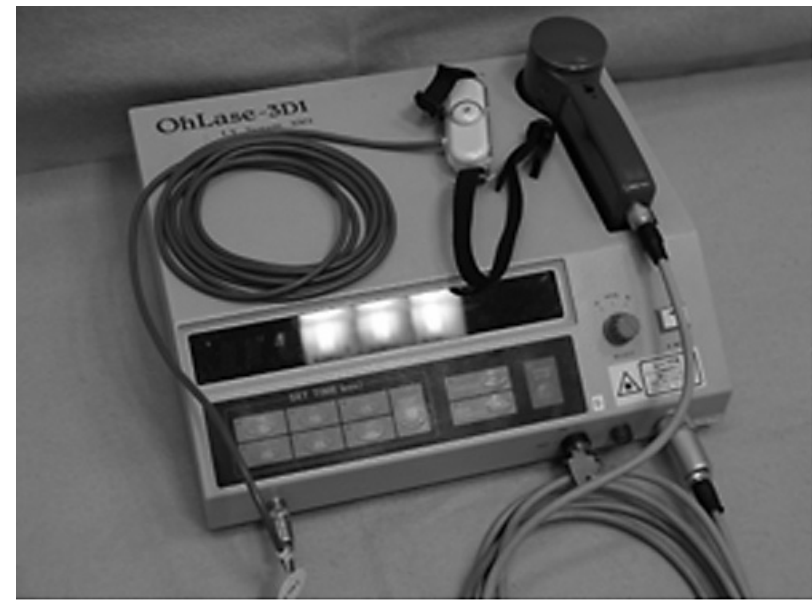

Fig. 1: The GaAlAs diode laser with the neck irradiator. The small probe attached to the coiled cable is the neck irradiator. The probe is placed somewhere along the posterior portion of the neck. The conventional hand held probe is located in its holding place on the right. The small nob below the hand held probe is the switch to change emission mode between the neck irradiator and hand piece and both.

in to the uterus, but cannot correct any individual pathological or other problems. In Japan, women often complain of constipation, stiff and sore necks and shoulders along with cold, clammy hands and feet. Such conditions are recognized as symptoms of poor systemic circulation and a high risk factor of becoming refractory to ART for infertility. In a questionnaire survey of female patients not attending our clinic for infertility but for other reasons, roughly $60 \%$ of them complained of the symptoms mentioned above. The same survey was performed for the patients being treated for infertility which resulted in $86 \%$ of the patients being symptomatic and their symptoms on the average were more severe than the general group (Table 2)

Originally LLLT for infertility started when an obstetrician experienced a sensation of warmth throughout his body after being treated with LLLT for lower back pain. This experience led to a study of LLLT as an adjunctive treatment for infertility. We retrospectively call this study the 'old project'. The transition of treatment modalities following the old project is summarized in Table 3. The old project was a study of patients at a single infertility clinic but the resulting pregnancy rate of $19.6 \%$ in a group of confirmed low responders to ART was so encouraging as to lead us to open the treatment of infertility for all patients attempting ART at other clinics. (1-2)

This project started on July of 1997 . No patients were refused treatment due to physical conditions of infertility or age. During this project, called the new 
Table 1: Comparison Between the New Project and Present (Neck Irradiator) Project

\begin{tabular}{lcc}
\hline & $\begin{array}{c}\text { New Project } \\
\text { Jul.2000- } \\
\text { Jan.31,2006 }\end{array}$ & $\begin{array}{c}\text { Present Project with } \\
\text { Neck Irradiator } \\
\text { Apr.1,2006-Jan.31,2007 }\end{array}$ \\
\hline \# of patients & 431 & 34 \\
Avg. age & 39.1 y.o $(26-52)$ & $41.7 \mathrm{y.o}$ \\
Avg. period of infertility & $4.5 \mathrm{yrs}$ & $3.9 \mathrm{yrs}$ \\
Avg.\# of ART & 8.4 & 6.8 \\
Avg.\# of LLLT & 16.0 & 10.5 \\
Pregnancy \% & $9020.9 \%$ & $38.8 \%$ \\
Live Birth Rate & $4853.9 \%$ & $0(0 \%)$ \\
\hline
\end{tabular}

Table 2: Prevalence of stiff necks and shoulders, cold clammy hands and feet (Of the 357 patients of the new project )

\begin{tabular}{lr} 
Sore shoulder only & 43 \\
Coldness only & 41 \\
Both sore and cold & 225 \\
Neither sore nor cold & 48 \\
\hline total & 357
\end{tabular}

\begin{tabular}{l}
\hline $\begin{array}{l}\text { No. of symptomatic } \\
\text { Patients }\end{array} \quad 309 / 357 \quad 86.6 \%$ \\
\hline
\end{tabular}

Table 3: Transition of Laser Treatment Modality for Female Infertility at Ohshiro Clinic

$\begin{array}{ll}\text { Old project } & \text { Oct. } 1996 \text { - Apr.2000 } \\ & \text { Direct application of laser to abdomen and lumbar area } \\ & \text { Treatment time: } 10-15 \text { min. }\end{array}$

Treatment time: $10-15$ min.
New project: Jul. 2000 - Apr.2006
Proximal priority treatment. Laser is irradiated mainly to the neck with stretch and local (abdomen and lumbar area ) treatment.
Treatment time: $30-40 \mathrm{~min}$.
Present project May $2006-$
PPT using the neck irradiator. Continuous laser irradiation to the neck. Direct application of laser to abdomen and lumbar area, two channel treatment if necessary.
Treatment time: $20 \mathrm{~min}$.

Table 4: Comparison of Pregnancy Rate by Age Group -New Project-

\begin{tabular}{|c|c|c|c|c|}
\hline & Pregnancy rate & Average \#of LLLT & Live births and rate & Miscarriage rate \\
\hline$<30$ y.o (9 pts) & $44.4 \%$ (4pts) & 6.5 times $(4-8)$ & $75.0 \%(3 \mathrm{pts})$ & $25.0 \%$ (1pts) \\
\hline $30-35$ y.o (88 pts) & $29.5 \%$ (26pts) & 12.6times $(4-61)$ & $80.7 \%$ (21pts) & $19.3 \%(5 p t s)$ \\
\hline 36 -38y.o (83 pts) & $31.3 \%$ (26pts) & 11.3times $(3-20)$ & $46.2 \%$ (12pts) & $53.8 \%$ (14pts) \\
\hline 39-40y.o (72 pts) & $16.7 \%$ (12pts) & 16.2times $(6-57)$ & $58.3 \%(7 \mathrm{pts})$ & $41.7 \%$ (5pts) \\
\hline $41-42 y . o$ (81pts) & $16.0 \%$ (13pts) & 11.5 times $(2 \sim 11)$ & $41.6 \%(5 \mathrm{pts})$ & $58.4 \%$ (8pts) \\
\hline$>43$ y.o (98pts) & $9.2 \%$ (9pts) & 16.3times $(4-40)$ & $22.2 \%$ (2pts) & $77.8 \%$ (7pts) \\
\hline total (431pts) & $20.9 \%$ (90pts) & 11.9times $(2-61)$ & $55.0 \%$ (50pts) & $44.0 \%$ (40pts) \\
\hline
\end{tabular}

project' there were some very dramatic cases such as a pregnancy and subsequent successful delivery of a child from a patient who was anovulatory for 15 years prior to presentation, ${ }^{(3-4)}$ or as in the case of a pregnancy and delivery in a women who had a history of pediatric malignancy and was treated with chemo- and radiotherapy. The side effect of that treatment had left her completely amenorrheic and the patient was seeking means for ovum donation abroad.

The results of the new project are shown in Table 4. The demographics of patients attending the Ohshiro clinic is quite different from other conventional ART clinics. Patients presenting to our clinic already have a number of failed attempts at other clinics and many come as 
a last resort. The high average age of the patients and long history of infertility treatment reflects this fact. Many patients have been turned down at other clinics for their age alone. It is quite obvious that a patient group of older women (around 40 years old) would be less successful in their attempt to bear a child than a group of younger (less than 33 years old) women. Our cumulative result of 90 pregnancies and the pregnancy rate of 20.9 $\%$ are noteworthy in a group where the projected pregnancy rate without LLLT could be as low as 5\%.

Since late 2006, the growing patient volume and limited manpower at Ohshiro clinic has necessitated a more efficient treatment where the same efficacy of treatment can be attained in a shorter treatment time. We were extremely careful to not to lower our standard of treatment efficacy due to this shorter treatment time. In order to do so we had to contrive a device that would continually deliver laser energy to the patient without a therapist holding it in place, in other words a hands-free treatment. Previously the therapist would have to hold the laser probe in place and turn on the switch of the laser only when the probe is in contact with the patients' body surface for safety reasons.. This meant that every time the probe was moved from point to point, the laser had to be switched off and the patient did not receive any laser energy until the therapist switched the laser on again. We requested the Japan Medical Laser Laboratory (JMLL) to develop new hardware to solve these problems, JMLL answered with a new device called the Neck Irradiator where the 60 $\mathrm{mW}$ GaAlAs laser probe was downsized and attached to a strap that could be placed around the patient's neck throughout the treatment session in a hands-free manner with continuous activation of the laser beam and irradiation of the target tissue made possible. This theoretically allowed the patients to receive the same total amount of laser energy in a shorter period of time compared to the previous treatment method.

The result of only 3 pregnancies in a group of 34 patients using the Neck Irradiator is somewhat disconcerting. Three more patients who have received treat-

\section{References}

1. OhshiroT, Fujii S, Sasaki K et al (2001): Laser therapy as an adjunctive treatment for severe female infertility. A preliminary report. Laser Therapy, 11 (2): 96-102

2. Ohshiro T, Inoue M and Kobayashi Y (1998): Low reactive laser therapy for refractory female infertility. Tokyo Journal of Obstetrics and Gynecology, 4 9 (3): 389-392.

3. Fujii S and Ohshiro T (2004): Low reactive level ment with both the previous method and this new method and hence are not included in this group, have become pregnant during the same period. An attempt at a logical explanation would be firstly the change in patient characteristics including the severity of infertility in this group. The average age of the patients using the neck irradiator has increased to 41.7 years old compared to the previous 39 .1years old. This may have had the biggest impact on the results. Another would be the difference in the accumulation of therapeutic effect. Previously, patients were advised to receive treatment at least once a week for the first ten weeks and then widen the period between treatments to once every other week. Many patients in this group, although they have continued treatments, have not fulfilled the regimen of one treatment per one week for ten weeks. Most of the women attending our clinic are working and perhaps could not spare the time for treatment. This may help explain the disappointing results and at the same time may show the importance of the time between treatment sessions.

Further studies are warranted in order to accurately assess the issue of the number of treatment sessions and the period between treatment sessions but from our experience using the Neck Irradiator, treatment has become more concise where the total amount of laser energy can be emitted to the patient in a shorter period of time without increasing the energy density of the laser beam itself. Overall patient satisfaction has increased despite the results. From a questionnaire survey of patients who have experienced both treatment styles, the most popular answer was that they were able to concentrate more on the treatment and relax , because they did not have to hold the laser hand piece for the therapist. The patients also felt that the treatment effect was as good or even better using the neck irradiator. The relatively small number of patients in this study may or may have not reflected the true efficacy of low reactive level laser therapy for the treatment of female infertility, and further compilation of data with a much larger patient set is required. laser therapy for low responding ovaries. In Modern Trends in Infertility Treatments, Ishiyaku Shuppan: 214-222.

4. Fujii S and Ohshiro T (2004): A case where low reactive level laser therapy was thought to be extremely effective in the treatment of female infertility. Tokyo Journal of Obstetrics and Gynecology, 53 (2): 207-211. 